\title{
Quelques remarques sur le projet d'une Histoire sociale des langues de France et les problèmes qui se sont posés lors de son élaboration ${ }^{1}$
}

\author{
Kremnitz, Georg \\ Institut für Romanistik, Universität Wien \\ georg.kremnitz@univie.ac.at
}

\section{De la genèse du projet et des buts qu'il se propose}

Quiconque s'intéresse au sujet des langues de et en France sait que le gouvernement Jospin tentait, vers la fin du siècle dernier, de signer et ratifier la Charte européenne des langues régionales ou minoritaires pour mieux intégrer le pays dans le courant de la politique européenne et en finir avec une exception française qui commençait à devenir gênante. Pour arriver à son but, le gouvernement fit établir plusieurs rapports (politique, juridique et finalement linguistique). C'est dans ce cadre que le philologue Bernard Cerquiglini a été chargé par les ministres de l'Education Nationale et de la Culture de rédiger un rapport sur l'inventaire des langues de France. La publication, en avril 1999, de ce rapport complété d'une liste des «langues de France », aujourd'hui communément appelé rapport Cerquiglini ${ }^{2}$, eut pour conséquence une nouvelle perception des réalités linguistiques complexes du pays. D'une conception de monolinguisme absolu, héritée, au moins comme idéal, de la Révolution, et constamment invalidée par les faits, ce rapport passe à une vision multilingue qui fait de la France le pays qui compterait le plus de langues en Europe occidentale ${ }^{3}$. L'on connaît la suite : la Charte fut bien signée par le ministre Pierre Moscovici, le 7 juin 1999 à Budapest, mais le président de la République de l'époque, Jacques Chirac, fit appel au Conseil constitutionnel pour obtenir un examen de la conformité de cette Charte avec la Constitution, examen à l'issue duquel le dit Conseil constitutionnel déclara cette Charte incompatible avec la Constitution française. Donc, la France demeure, avec la Grèce et la Turquie, un des pays qui ne l'ont toujours pas ratifiée. Il n'est pas de mon propos ici d'épiloguer sur les éléments de politique intérieure qui ont joué un rôle dans cette démarche ${ }^{4}$. On peut cependant regretter que la France n'ait pas saisi cette occasion pour sortir d'une situation de plus en plus incommode.

Toutefois, l'existence de la liste Cerquiglini a ouvert de nouvelles possibilités de réflexion et innové dans la manière de voir les choses. C'est dans ce contexte que naquit l'idée de lancer un projet qui tenterait de décrire les comportements langagiers des habitants de la France à travers l'histoire, en mettant l'accent sur les rencontres des langues dues à des raisons très diverses ${ }^{5}$. Il était clair d'emblée qu'un tel projet devait innover sur plus d'un point.

Permettez-moi d'abord d'esquisser les contours de l'idée et du but à atteindre, de dire quelques mots sur le fonctionnement du groupe de travail qui s'est formé avant de relever quelques points critiques de la recherche antérieure et d'indiquer quelques innovations, puis d'en arriver à quelques remarques sur les choix que le collectif a effectués. Ensuite, j'esquisserai le plan sans doute définitif de la publication, avant d'en arriver à quelques perspectives plus générales aussi bien sur le plan scientifique que sur le plan social.

La conséquence immédiate de mon échange de courrier avec M. Cerquiglini fut l'organisation d'un colloque qui se tint à l'INALCO les 30 septembre et $1^{\text {er }}$ octobre 2004, avec le soutien de la DGLFLF (une soixantaine de personnes participèrent aux débats) ${ }^{6}$. L'idée reçut beaucoup d'encouragements, mais certains collègues insistèrent sur les difficultés d'un semblable projet. Aujourd'hui, je les écouterais sans doute d'une oreille plus attentive qu'alors. J'avais en effet très certainement sous-estimé les problèmes qui allaient se poser.

A ce moment-là, un collectif d'universitaires et de chercheurs se forma afin de piloter le projet; il se compose aujourd'hui de Carmen Alén Garabato (Montpellier), Klaus Bochmann (Leipzig/Halle), Henri 
Boyer (Montpellier), Fañch Broudic (Brest), Dominique Caubet (Paris), Marie-Christine HazaëlMassieux (Aix-en-Provence), Georg Kremnitz (Vienne), François Pic (Toulouse) et Jean Sibille (à l'époque Paris, DGLFLF, aujourd'hui Toulouse CNRS), le secrétariat en a été assuré tout d'abord par Christiane Böck (Vienne), et depuis la fin 2008 Carmen Alén Garabato en assume la charge ${ }^{7}$. Ce collectif s'est réuni régulièrement deux à trois fois par an, la dernière réunion ayant eu lieu fin janvier dernier, et nous espérons que la prochaine coïncidera avec la présentation du résultat final au public. Nous sommes actuellement à la fin d'une phase de relecture des textes reçus et de mise en forme de l'ensemble. Mon idée initiale était l'élaboration d'une sorte d'encyclopédie en plusieurs volumes, mais peu à peu, le principe de réalité a gagné du terrain, et il a fallu que je m'avoue que pour parvenir à un telle présentation les travaux préparatoires manquaient très souvent, mais également que les besoins financiers dépassaient de loin ce que nous pouvions espérer réunir. D'ailleurs, le collectif dans son ensemble hésita entre deux solutions : l'une, plus complète, d'environ 5 millions de signes et l'autre, plus légère, de seulement 2 millions de signes. Nous avons finalement dû opter pour cette dernière, surtout guidés par le souci de présenter les résultats de notre travail dans des délais raisonnables. Actuellement, nous nous acheminons vers un compromis, avec près de 3 millions de signes.

De quoi peut-il donc s'agir ? Je dirais de manière tout à fait provisoire, qu'il y est traité essentiellement de l'évolution des formes de communication à l'intérieur des différents groupes linguistiques, mais, en même temps, des rapports que les locuteurs de formes linguistiques différentes établissent entre eux et avec la langue dominante, le français. Le français est la première des langues de France ; et si les histoires de la langue française (qui évoquent en général les formes de la communication, quoique assez - voire trop - rapidement) sont nombreuses, il est parfois surprenant de voir le peu de détails dont nous disposons sur l'emploi même de cette langue à travers l'histoire. Mais il ne s'agit pas ici, en premier lieu, de nous occuper de l'histoire de la communication en la langue que nous connaissons le mieux, mais de l'histoire de toutes les autres langues ou variétés qui sont obligées, à certains moments historiques, d'entrer en contact avec cette langue dominante et entre elles également; il s'agit en outre de donner à voir, dans la mesure du possible, une architecture complexe de la communication dans son évolution. Le jeu est donc multiple, il le devient encore davantage, quand nous pensons que beaucoup de langues de et en France ont des rapports au-delà des frontières; elles peuvent recevoir des impulsions importantes de l'extérieur. Si l'on accepte une idée de totalité, dans un sens que le marxisme - surtout Georg Lukács ${ }^{8}$ - a emprunté à l'idéalisme allemand (ce sens apparaît initialement chez Winckelmann dans son Histoire de l'art antique $(1764)^{9}$, on entrevoit l'ampleur du champ. Il ne faut pas perdre de vue que le terme de communication renferme beaucoup d'éléments tant externes, comme la conscience des locuteurs, le statut des langues, les degrés respectifs de normativisation (codification) et normalisation (emploi social) ${ }^{10}$, qu'internes comme tous les phénomènes des changements linguistiques.

Je voudrais rapidement évoquer deux problèmes pratiques auxquels le collectif a été confronté. Le premier est celui de la dispersion des membres du collectif sur trois pays. Ils travaillent dans sept villes différentes et ils sont tous des chercheurs actifs qui fonctionnent dans des contextes multiples. De sorte qu'il n'a pas toujours été facile de se réunir, et certains processus de rapprochement théorique et méthodologique ont pris davantage de temps qu'il aurait été souhaitable. Ainsi, il est parfois devenu difficile de maintenir à son plus haut niveau l'enthousiasme de certains collaborateurs potentiels tel qu'il s'était manifesté lors de la rencontre de 2004. Le second problème est celui des finances. Certes, le collectif a obtenu à plusieurs reprises des subventions de fonctionnement de la DGLFLF et au départ aussi de l'Université de Vienne. Par ailleurs, deux de membres de notre collectif, Henri Boyer et Carmen Alén Garabato, organisèrent un colloque à Montpellier en décembre 2006, intitulé "Les langues de France au $\mathrm{XXI}^{\mathrm{e}}$ siècle. Vitalité sociolinguistique et création culturelle ${ }^{11}$ qui procura une occasion unique de rencontre et de discussion. Ainsi, le collectif a pu assurer les progrès des travaux préparatoires. Cependant, les dépenses les plus importantes nous attendent encore: la publication certes, mais auparavant tous les travaux de relecture, d'harmonisation, de correction et de formatage ne pourront se dérouler sans des subventions substantielles. C'est dans ce contexte que la composition du collectif se révèle être peu avantageuse. Car il va de soi que le gros de moyens pour la publication devrait venir de la France. Mais le principal responsable du collectif ne travaille pas en France, il est donc mal placé pour y obtenir des subsides. Inversement, les autorités autrichiennes ne s'intéressent guère au sujet - l'idée 
européenne ne perce pas encore à ce point. Il n'est pas impossible d'obtenir des soutiens de l'Europe, mais seulement quand on dispose déjà des subventions «nationales». Ce sont donc les membres français du collectif qui doivent préparer le gros des demandes de subventions, sans disposer des infrastructures nécessaires en principe à ce dessein. Je sais bien que ce problème ne concerne que ce projet, mais je pense qu'il serait urgent que la communauté scientifique s'en saisisse, car l'organisation actuelle de la recherche qui de plus en plus repose sur des épaules privées - ou du moins non-étatiques - rend nécessaire la création de structures, universitaires ou autres, qui disposent des connaissances pour élaborer ces demandes, devenues de plus en plus compliquées. En principe, le temps des chercheurs, qui ne sont pas formés à cela et desquels le rendement est par conséquent discutable, devrait être trop précieux pour être " gaspillé » de cette façon. Tout de même, le collectif a entrepris un ensemble de démarches et espère ainsi atteindre le but ultime de ses efforts.

\section{Les motivations du collectif et quelques tentatives d'innovation}

Vouloir montrer l'histoire et l'évolution de la communication sur un terrain où une multitude de langues se parle signifie d'abord privilégier ce qu'on appelle communément l'histoire externe, non pas d'une seule langue mais d'un réseau compliqué de formes de communication, tout en montrant que la séparation quelque peu artificielle entre histoire externe et interne a tout au plus un droit méthodologique à l'existence. En réalité, les deux sont intimement et dialectiquement liés. Cela implique entre autres une vue nouvelle sur le jeu entre production et réception langagière.

\subsection{Rééquilibrer les relations entre production et réception linguistiques}

Il fallait par conséquent remettre en question l'attention trop exclusive consacrée à la production langagière de la part de la recherche ${ }^{12}$. Je m'explique : la pratique d'une langue sert deux buts à la fois : d'une part la communication, de l'autre la démarcation. Quelle que soit la variété linguistique dont j'use, je m'en sers à la fois pour communiquer avec d'autres, mais je m'inscris en même temps dans mon ou mes cercles de communication et je me démarque de tous les autres. Cette distance peut être minime et ne pas entraver la communication courante, mais elle peut aussi être (presque) totale. Dans ce cas nous disons ne pas comprendre l'autre. Mais, en réalité, notre communication est toujours fragmentaire; on peut essayer de la rendre plus complète, mais finalement elle reste fuyante, nous le savons au moins depuis les travaux de Sigmund Freud (de même la démarcation n'est jamais totale, on l'oublie souvent). Le fait que nous ne sommes jamais sûrs du degré de communication réellement obtenu (pensons aux messages contradictoires) rend la réception et l'interprétation des signes reçus, bref la compréhension, toujours un peu incertaine, nous le savons. Des deux fonctions, la première, la communication, est primordiale. On n'entre pas, sauf en de rares exceptions qui paraissent extrêmement construites, en communication pour ne pas communiquer. La communication s'établit d'abord entre un émetteur et un récepteur; elle peut se compliquer, par la multiplication des représentants des deux pôles ou par l'intervention d'éventuels intermédiaires: elle peut connaître des distorsions par d'autres influences externes. Elle se fait nécessairement toujours avec les seuls moyens communicatifs que les interlocuteurs ont à leur disposition. Certes, là où elle devient habituelle, des traditions de comportement se créent ; la géographie linguistique par exemple les a mises en évidence. Mais ce sont des faits de communication qui disparaissent dès que les raisons de cette communication n'existent plus. La relation créée entre une langue et un territoire est une création relativement récente, liée avant tout au succès du nationalisme moderne - il est vrai que les langues de pouvoir apparaissent plus tôt, mais, au départ elles ne revendiquent généralement pas l'exclusivité territoriale qui depuis le $\mathrm{XIX}^{\mathrm{e}}$ siècle devient la norme théorique (mais qui nulle part n'arrive à devenir la réalité sociale, du moins jusqu'à présent).

La linguistique, depuis le renouveau de ses méthodes au début du XIX ${ }^{\mathrm{e}}$, s'est intéressée presque exclusivement à la production langagière, je l'ai dit plus d'une fois. Elle n'a guère tenu compte de la réception des sons émis par un auditeur, elle a presque complètement négligé le jeu dialectique de la communication. Pendant le $\mathrm{XIX}^{\mathrm{e}}$ siècle, ce comportement était relativement compréhensible, car il n'existait pas encore de moyen technique pour conserver la production langagière parlée. L'unique 
possibilité de conserver du matériel linguistique était l'écriture. Ceci donnait à l'écriture une importance pratique qu'elle n'a d'ailleurs, de nos jours, pas entièrement perdue. La langue à laquelle nous nous référons mentalement continue à être, du moins pour le commun des usagers, et dans la plupart des cas, la langue écrite. De cette manière, les règles de la production écrite sont devenues les règles les plus importantes pour la praxis sociale, et les linguistes n'ont pas pu vraiment changer les données. Beaucoup d'entre eux ont les mêmes réflexes, surtout quand ils se doublent de grammairiens. Or, la langue ainsi interprétée est le fruit d'une double réification des processus de communication, car d'une part elle retient de l'acte de communication l'abstraction langue et de l'autre elle réduit cette langue à langue écrite, une forme encore plus réduite du phénomène observé qui suit des normes très strictes et restrictives. Le concept ainsi obtenu ne s'intéresse qu'à une partie du phénomène en question, il fait abstraction d'éléments essentiels.

Si au XIX ${ }^{\mathrm{e}}$ siècle, cette double limitation est compréhensible comme conséquence des moyens techniques disponibles, il est plus surprenant de voir que la linguistique du $\mathrm{XX}^{\mathrm{e}}$ ne change guère d'orientation. Les néogrammairiens qui dominent la discipline encore pendant la première moitié du siècle, ne pensent qu'à la production langagière. Leurs lois phonétiques ne prennent en considération que l'émission. Comme ces lois ne suffisent pas pour expliquer les faits, ils inventent l'analogie comme deuxième motif des changements phonétiques, ils parlent de formes directrices, mais ils ne se posent pas vraiment la question du pourquoi de l'existence de ces formes. Au prix d'un seul pas supplémentaire, ils auraient pu voir qu'il s'agissait de questions de communication et que la réception potentielle y jouait un rôle important. Le structuralisme naissant ne se concentre plus sur la diachronie et découvre les fonctionnements de la synchronie, mais il le fait presque uniquement du côté de la production, alors que le Cours de Saussure insiste sur les différences entre les aspects individuel et social du langage. Le Cours examine en détail les conditions de production langagière, mais la réception et la communication restent les parents pauvres ${ }^{13}$. Il distingue entre langue et parole, une différenciation qui aurait pu être un chemin pour voir les problèmes de communication, mais le terme même de communication n'apparaît pas dans l'index du livre. La plupart des successeurs de Saussure suivront la même voie, négligeant même les progrès du côté social que le Cours contient. Et même la dialectologie et la géographie linguistique qui étaient les plus proches des phénomènes de communication, n'en tiennent guère compte, à quelques exceptions près, parfois notables cependant comme Jules Gilliéron ${ }^{14}$.

C'est ainsi que la linguistique présentera relativement tard des modèles de communication, précédée en cela d'ailleurs par la nouvelle technologie de la radio. Quand Karl Bühler proposera en 1934 son Organon-Modell ${ }^{15}$, ce sera le premier grand essai du côté de la linguistique (Bühler était cependant avant tout psychologue), mais il privilégie encore la production (comme le sous-titre l'indique). Et Roman Jakobson, l'autre linguiste pionnier dans ce domaine, fait encore de même en $1960^{16}$. Or, entre temps, en 1949, Shannon et Weaver, représentants d'une toute nouvelle discipline, la cybernétique, qui met la communication au centre de ses préoccupations, avaient présenté leur modèle de la communication ${ }^{17}$. Ce schéma ornera pendant longtemps tous les manuels de linguistique, et il est toujours l'ancêtre des modèles que l'on voit aujourd'hui. Mais la praxis des linguistes n'a guère changé. C'est sans doute dû, en partie, à l'attitude du courant le plus puissant de la linguistique actuelle, le générativisme. Son fondateur, Chomsky, a dit plus d'une fois que la communication n'avait pas d'importance accrue pour la discipline. Or, je pense que cela est une erreur.

La limitation de l'analyse à la production langagière peut se comprendre sans difficulté en tant que stratégie de recherche précise, mais si les sciences du langage négligent systématiquement les données communicatives, elles se privent simultanément des possibilités de comprendre et interpréter la production. Car même celle-ci s'établit dans un jeu dialectique complexe entre production et réception, et ne pas en tenir pas compte équivaut à une auto-restriction sérieuse. Par contre, une nouvelle approche de la grammaire historique qui tiendrait davantage compte des aspects communicatifs, pourrait mener, ici et là, à des résultats nouveaux voire surprenants. Il est clair que cette lacune apparaît plus facilement dès qu'on se tourne vers les fonctions sociales du langage. C'est pourquoi il me semble essentiel qu'une histoire de la communication en France apporte non seulement des connaissances supplémentaires pour le sujet envisagé, mais aussi des avancées pour la théorie de la discipline. 


\subsection{Dénationaliser les histoires de la communication}

Un autre point délicat de la recherche antérieure est celui du nationalisme implicite de la plupart des histoires existantes : presque toutes les histoires de langues sont des histoires d'une langue, comme si les langues fonctionnaient de manière isolée. Dans ces textes, une langue, le protagoniste, se trouve au centre de l'observation, soit, dans le cas des langues dominantes, en tant qu'histoire d'un succès, d'une imposition, soit au contraire, dans le cas des langues dominées, en tant qu'histoire de la persécution et réduction de cette langue à son existence résiduelle dans l'actualité. Cela est sans doute une conséquence (implicite) de la vision du nationalisme européen du $\mathrm{XIX}^{\mathrm{e}}$ siècle qui considère l'homme comme un être naturellement monolingue (et pour qui, soit dit en passant, le plurilinguisme est une source de malheur psychique $^{18}$ ). Je ne peux développer ici l'évolution de cette conception, qui, dans un certain sens, naît d'un mariage malheureux de la conception politique de la nation (formulée au départ surtout par Montesquieu $^{19}$ et élargie sous la Révolution à l'ensemble de la population masculine, repris plus tard par Renan) avec la conception culturelle qui considère la langue come un élément essentiel d'un groupe humain (comme on sait, cette conception remonte à Herder, mais elle a, dans les mouvement des guerres révolutionnaires, profondément changé de contenu). Selon cette conception, la langue apparaît comme un élément constitutif et inné de l'être du locuteur. La pratique du nationalisme à partir du XIX ${ }^{\mathrm{e}}$ siècle a associé les deux : une nation dispose d'une seule langue (et d'un Etat), ceux qui à l'intérieur de cette nation parlent volontairement une autre langue sont pour le moins suspects; d'autre part, un groupe qui s'auto-définit comme nation doit par conséquent aspirer à former un Etat indépendant pour y suivre les mêmes règles d'inclusion/exclusion. En plus, ce nationalisme rattache le plus souvent une langue à un territoire ou tout au moins à un espace (les changements d'espace d'une langue se comptabilisent alors le plus souvent en « victoires » ou en « défaites »). Or, depuis un certains temps, nous avons réappris que les espaces vraiment monolingues sont l'exception (pour ne pas dire qu'ils sont inexistants de nos jours), que la migration est une pratique qui traverse toute l'histoire de l'humanité et que sous la dénomination constante d'un groupe, sous la surface et l'apparence de cette domination, des phénomènes très complexes comme l'assimilation, la dissimilation, voire des cas de figure encore plus compliqués peuvent se produire. Et puis, les individus ne sont pas naturellement monolingues. Les langues coexistent toujours - sauf exceptions rarissimes - et elles se trouvent en contact et en conflit. En général, il n'y a pas seulement deux groupes et deux langues en contact, mais les situations peuvent être bien plus complexes, avec des partenaires qui entrent et qui sortent de la scène, comme dans une pièce de théâtre un peu surchargée.

Il va de soi que cette banalité dans le domaine des sciences du langage que je viens d'évoquer devrait avoir des conséquences pour les théories et les praxis politiques.

Naturellement, s'il est justifié, en théorie et en méthode, d'isoler un objet afin de l'observer, il faut néanmoins tenir compte du fait qu'il n'est qu'élément d'un ensemble plus vaste dont tous les éléments interfèrent dans la communication. Si l'on considère la perspective isolationniste comme absolue, on arrive à des distorsions considérables. C'est pourquoi je pense qu'il est important de mener, à côté de recherches sur des histoires de communications de sociétés considérées séparément, des travaux qui prennent en considération des territoires, voire des espaces, et qui montrent les jeux complexes de la communication à entrées multiples. Si un jour nous possédons un certain nombre de travaux de ce type couvrant des espaces complexes et prenant en compte des changements dans une profondeur diachronique considérable, nous pourrons alors parler d'une véritable sociologie historique de la communication. Personnellement il me semble qu'il serait important de parvenir un jour à présenter un tel tableau complexe de l'histoire de la communication en France.

\subsection{Repenser les différences entre histoire de la langue et grammaire historique}

Une troisième préoccupation résulte du fait que, très souvent, les ouvrages existants constituent une sorte de compromis entre une grammaire historique et une histoire externe de la langue en question. Souvent, les conceptions ne sont pas claires. Les grammaires historiques tendent à présenter des états successifs 
d'une langue, mais presque toujours de manière statique, sans pouvoir montrer les changements des conditions de communication qui ont abouti à ces changements. Certes, il y a les tendances contradictoires à l'économie et à l'expressivité ${ }^{20}$ que nous connaissons tous, mais les évolutions des langues prouvent bien qu'il ne s'agit pas que de cela. Les néogrammairiens ont commencé à décrire les mouvements, mais ils se sont surtout basés sur les périodes où les charges idéologiques des langues étaient faibles, l'époque moderne fait voir que les motivations favorisant des changements linguistiques se sont multipliées. D'une manière plus ou moins complémentaire, les histoires classiques des langues présentent bien les changements politiques et sociaux, mais elles n'entrent que rarement dans les conséquences communicatives ni même linguistiques de ces évolutions. Des considérations multifocales sur les changements des conditions de communication ne se rencontrent guère, si l'on fait abstraction des plus grandes révolutions comme par ex. l'introduction de l'imprimerie. Il serait cependant nécessaire de montrer comment des changements dans les sociétés - qui parfois peuvent paraître relativement insignifiants - ont entraîné ou tout au moins favorisé des changements de la communication dans ces sociétés. Pour ce faire, je crains qu'il faudrait employer des modèles de communication bien plus complexes que ceux qui se trouvent dans des manuels d'histoire des langues et qui, le plus souvent, se limitent à observer les couches sociales les plus aisées dans les capitales respectives. Il ne fait pas de doute que ces groupes sont le plus souvent les modèles que les autres couches imitent, mais ils se trouvent dans des relations complexes avec les habitudes communicatives d'autres couches sociales que, fréquemment, les histoires des langues ne prennent même pas en compte. La concentration trop exclusive sur les élites bloque facilement la vue sur les mouvements complexes des sociétés. Il est vrai que des travaux de ce type nécessitent l'utilisation de modèles plus complexes de sociétés que ceux employés en général ; il est possible qu'ils demandent des efforts si grands qu'ils ne sont réalisables que de manière ponctuelle. L'observation rigoureuse des changements des nécessités de la communication pourrait cependant prodiguer des éléments supplémentaires pour comprendre les mouvements des sociétés et inversement. Ces travaux pourraient peu à peu rendre superflue la différenciation entre histoire interne et externe des langues, car elles pourraient établir, avec bien plus de précision que jusqu'à présent, les influences mutuelles des deux domaines, société et communication.

Il faut dire que ces dernières décennies ont enregistré des progrès, tout au moins pour certaines langues. Plusieurs histoires sociales de langues, allant dans le sens que je viens d'esquisser, ont été publiées, depuis un quart de siècle environ, si je ne m'abuse ; cependant, elles restent des exceptions et elles ont des objectifs et des contenus parfois très divers ${ }^{21}$. Une recherche bibliographique - qui ne peut pas être exhaustive - ne donne que peu de titres explicites dans ce domaine. Il est bien évidemment souhaitable que leur nombre augmente. ${ }^{22}$

\section{Quelques indications sur les choix que le collectif a effectués}

\subsection{Généralités}

Précisons d'abord que le collectif Pour une histoire sociale des langues de France pense que l'histoire de la communication a connu - non seulement en France - deux moments de mise en question profonde et de renouveau : à la Renaissance avec l'introduction de l'imprimerie et l'avènement des langues des cours supplantant définitivement, dans un processus multiséculaire, le latin, et à la Révolution française qui a préconisé le monopole de la communication en français et qui a doté les langues en Europe d'une nouvelle charge idéologique. Actuellement, nous vivons une troisième révolution des données de la communication, où une oralité renouvelée tend à reprendre, avec des modifications importantes et jusqu'à présent difficilement saisissables, une place que le pouvoir de l'écrit lui avait enlevée ${ }^{23}$. S'il ne néglige pas les étapes antérieures de la communication, le collectif est conduit, tant par la disponibilité des sources que par l'importance accrue de connaissances pour nourrir l'actualité, à privilégier la période contemporaine, de la Révolution à nos jours.

Cela veut dire qu'il a fallu jeter un regard critique sur les différents termes que nous utilisons - souvent sans nous rendre compte des contradictions internes qu'ils renferment - afin de nous mettre d'accord sur 
les définitions employées dans l'ouvrage. Cela vaut pour des termes aussi répandus et pourtant aussi peu clairs que le mot «langue $»{ }^{24} \mathrm{La}$ linguistique l'a bien défini, la sociolinguistique aussi, mais les deux définitions ne coïncident pas, et l'usage social diffère encore. Il faut, au moins dans l'espace d'un livre, lever ces contradictions. On pourrait nous objecter que dans un projet semblable au nôtre il n'est pas nécessaire de réinventer la roue. Mais ces questions de définition ainsi que les différences entre les définitions se répercutent dans les pratiques même de la communication. Elles deviennent souvent acteurs dans notre champ de travail. Un regard critique sur elles s'avère par conséquent indispensable.

Enfin, tant par souci d'économie que de clarté, il a été tenu compte du fait qu'un nombre important de données de la communication concernent la France entière; ces sujets-là sont traités en des chapitres communs et sous l'angle de « questions transversales » ou « générales ». D'autres problèmes demandent un traitement spécifique par langue ou communauté communicative ; le collectif entend éviter à tout prix que le produit final ne soit qu'une accumulation, une juxtaposition de monographies reproduisant des données existantes.

\subsection{Le cadre territorial et la répartition des langues}

Le projet étant issu, dans un certain sens, comme je l'ai déjà indiqué, de la publication de la liste Cerquiglini, il semble naturel qu'il en adopte le cadre territorial, à savoir la France actuelle, incluant les DOM et les TOM. De même, beaucoup de langues comptant des locuteurs au-delà des frontières, il doit être tenu compte de ce fait. Il va de soi, en outre, que leurs relations diffèrent fortement entre elles.

Les différentes versions de la liste Cerquiglini ne coïncident pas totalement en ce qui concerne la répartition des langues concernées. Le collectif a donc décidé de privilégier une classification commode, établie sur les critères de leur insertion dans l'espace communicatif français. Il y a, d'une part les langues minoritaires autochtones que l'on appelle aujourd'hui généralement d'un terme que je considère peu réussi les «langues régionales » ${ }^{25}$; il y a, d'autre part, les langues de l'outre-mer, héritages de l'aventure coloniale française ; et il y a enfin les langues de l'immigration, récente ou ancienne, ces langues n'ayant en général pas d'assise géographique précise en France. Une place à part doit naturellement être faite à la langue des signes française (LSF) puisqu'elle remplace le son par le geste. Il faut cependant considérer cette classification, pourtant pratique, avec une certaine circonspection: que faire des locuteurs de l'occitan qui se sont déplacés, par exemple à Paris ou dans le Nord ? des Antillais qui sont venus en France métropolitaine et qui ont parfois commencé, à ce moment-là précisément, à s'intéresser à la langue et à la culture créoles ? Historiquement, la France a «grandi » : il faut ainsi, dans les Pyrénées-Orientales ou en Alsace, comme en Flandres, tenir compte des relations de communication antérieures au rattachement de ces territoires à la France. Sur un point, la liste Cerquiglini ne donne pas d'image fidèle de la communication : c'est celui des langues de l'immigration. Nous savons bien que dans ce domaine les décisions sont difficiles à prendre. Un des critères de l'intégration à la liste a été la condition que les langues prises en considération ne devaient pas avoir de statut officiel ailleurs ${ }^{26}$. Ce critère a provoqué l'exclusion de langues de la liste de langues dont de nombreux locuteurs ont laissé leurs traces en France ou continuent à parler leurs langues d'origine, comme l'italien, le castillan ou le polonais par exemple. Du point de vue de l'histoire de la communication en France, il ne semble pas possible de suivre la liste sur ce point. Le collectif a par conséquent fait sienne la différenciation que l'on peut déjà lire parfois entre « langues de France » et « langues en France ».

Or, les langues en France sont celles qui actuellement posent les plus grands problèmes. D'une part, l'exhaustivité dans ce domaine semble impossible, des microgroupes pouvant s'installer et communiquer dans leurs langues respectives sans que la société environnante s'en rende vraiment compte ; en outre, souvent la mobilité est très grande et la durée du séjour de certains groupes limitée. Ce champ des langues en France est donc extrêmement mouvant et nécessiterait une observation continue ${ }^{27}$. Il faut ajouter que dans de nombreux cas il n'est guère possible de trouver des spécialistes qui auraient pu donner même une impression plus ou moins succincte. D'autre part, une énumération aussi complète que possible des groupes vivant sur le sol de la France aurait été souhaitable. En naviguant entre ces difficultés le collectif 


\begin{abstract}
s'est décidé à chercher une solution médiane qui ne tend pas à l'exhaustivité dans ce domaine et se contente dans certains autres cas d'articles ou de fiches de synthèse. Pour d'autres langues, il n'a pas été possible de trouver de collaborateur. Il est vrai que cette décision est une concession à laquelle je ne me suis personnellement pas résigné de gaîté de cœur.
\end{abstract}

\title{
3.3 L'architecture de l'ouvrage prévu
}

La publication est arrêtée en quatre grandes parties, inégales entre elles. Comme déjà indiqué, une première partie traite les questions transversales ou générales qui concernent l'ensemble de la France et des communautés communicatives. Elle sera suivie de trois parties, traitant successivement les langues de la France métropolitaine (autochtones), les langues des DOM/TOM, et finalement les langues issues de l'immigration. A l'intérieur de chacune de ces trois dernières parties, une première sous-partie traite à son tour les questions générales concernant cette partie, puis une partie monographique s'occupe des différents aspects des langues de ce domaine. Il va de soi qu'un système de renvois doit éviter des doublons, surtout dans des cas où une communauté communicative relève de plusieurs chapitres.

La première partie, consacrée aux questions générales, a pour objet la clarification de la terminologie employée, ainsi que l'évolution des différentes dénominations adoptées pour un même objet. D'autre part, elle doit rendre visibles les relations entre la pratique communicative et des appartenances à certains groupes, qu'ils soient religieux, politiques ou autres. Il y faut aussi prendre en considération les forces sociales et les institutions qui ont réglé (et règlent encore) la communication : l'école, l'administration, mais aussi la législation, etc. Parmi elles, les instances normatives de la langue, l'installation d'une norme prescriptive et sa généralisation successive par l'école jouent un rôle particulier. Aujourd'hui, les massmédias relayent voire remplacent l'école, et certaines formes externes du langage qui naguère étaient hautement valorisées perdent relativement d'importance ; il suffit de penser à l'orthographe. Il faut voir si cela concerne toutes les langues ou si seuls certains groupes communicatifs ont connu ce déplacement. Le collectif porte une attention particulière au rôle du chercheur qui, d'un côté, est observateur des processus de communication qui se déroulent dans la société, mais, de l'autre, prend part aux pratiques et aux idéologies langagières de cette société ; il doit par conséquent faire très attention à ne pas mélanger ces deux rôles. Ceci devient plus important encore pour des groupes où les chercheurs jouent un rôle social avéré pour le maintien ou la reconquête de pratiques communicatives menacées.

La partie consacrée aux langues autochtones dominées doit, dans son approche transversale, réserver une place aux évolutions de la communication dans la France métropolitaine. Elle observe, avec un soin particulier, les réglementations que l'Etat impose à la communication et les effets que ces règlements ont eus et continuent à avoir. Il faut surtout prêter attention aux rapports asymétriques entre le français et ces langues, les éléments de répression linguistique (qui ne sont rien d'autre qu'un effort d'intimidation sociale et donc à terme de silence), et, plus tard, les modestes tentatives d'insertion de ces langues dans les cursus scolaires et universitaires. Dans la partie monographique, il faut préciser les détails qui concernent les différents groupes en particulier. D'une part, il s'agit d'une histoire de la communication à l'intérieur d'un groupe, mais aussi de ses rapports avec le monde externe, que celui se trouve à l'intérieur d'un même Etat ou qu'il soit transfrontalier. Nous nous trouvons là dans un jeu dialectique entre la conscience et les comportements du groupe considéré et les conceptions qui viennent de l'extérieur, le plus souvent de l'Etat ou de ses représentants. Ce jeu ne serait pas possible sans l'idéologisation que la communication et particulièrement les langues ont subie depuis le début des processus révolutionnaires $\mathrm{au} \mathrm{XIX}^{\mathrm{e}}$ siècle. Sans cette charge idéologique les mouvements de renaissance n'auraient pas été imaginables, ni les tentatives de faire de ces formes de communication de véritables langues dotées de tous les outils qui ont donné aux langues dominantes leur position. Les groupes de communication sousprivilégiés ont ainsi adopté les processus de double réification dont j’ai déjà parlé (cf. 2.1.). Toutefois, ce qui est remarquable, c'est qu'en France métropolitaine aucun de ces groupes n'a réussi, à ce jour, à imposer son point de vue, contrairement à beaucoup d'autres pays en Europe qui ont éclaté ou ont dû faire des concessions considérables aux groupes communicatifs périphériques. C'est même aujourd'hui devenu la norme politique en Europe $^{28}$. 
Les parties concernant les DOM/TOM et l'immigration suivent, en gros, le même schéma, avec les modifications qui s'imposent. Ces modifications concernent les formes de l'intégration de ces terres au pouvoir français et les conséquences qui en découlent pour la communication. Dans une partie des actuels DOM, les conditions pour une communication ont pratiquement dû être recréés, à cause de l'annihilation presque complète de la population antérieure ${ }^{29}$, et nous nous trouvons devant des situations très particulières de genèse de nouvelles formes de communication - les langues créoles en sont les témoins. Dans d'autres cas, le manque de sources et les relations anciennes entre différents groupes de populations communiquant de diverses manières entre eux, a rendu nécessaire un traitement non pas monographique mais qui a pris en compte l'ensemble ou des sous-ensembles des groupes communicatifs présents. Les choses se compliquent encore quand on se tourne vers les langues de l'immigration. Comme je l'ai déjà dit, on y fait la distinction entre langues de France et langues en France, différence qui pour notre propos ne joue qu'un rôle mineur. Parmi les langues de France issue de l'immigration, il y a des langues qui depuis très longtemps sont pratiquées sur le sol français, comme le yiddish, le judéo-espagnol ou le rromani ; d'autres sont arrivées plus récemment comme l'arabe maghrébin, le berbère (tamazight) ou l'arménien (occidental). Mais, pour en rester à l'exemple du yiddish, il semble aujourd'hui impossible de ne prendre en compte que cette langue des communautés juives de la France, sans tenir compte des autres formes de communication qu'elles utilisent (en réalité plus souvent). Certes, les traditions des différents groupes juifs divergent, mais depuis un certain temps ils coexistent dans une société étatique, ils règlent leurs comportements communicatifs et ils tentent de communiquer entre eux et avec l'extérieur en conséquence. Dans ce cas, il semble préférable de prendre en considération l'ensemble du groupe pour pouvoir rendre compte également des contacts internes. La situation est quelque peu comparable en ce qui concerne les immigrés de l'Afrique du Nord qui à leur tour ont plusieurs modes de communication à leur disposition et qui les utilisent (ou abandonnent) selon des critères souvent très complexes.

\section{Conclusion}

Le but de notre initiative est de donner pour la première fois aux spécialistes des sciences du langage et de la communication et au public cultivé francophone une vision aussi complète que possible de l'histoire de la communication dans ce pays en y intégrant des aspects qui ne sont, en général, pas pris suffisamment en considération. Notre projet veut innover du point de vue de la théorie comme de celui de la méthode. Comme il n'existe, à ce jour, pas de projet comparable réalisé, il pourrait servir de modèle. Il aimerait insister sur le fait que les sciences du langage doivent s'intéresser beaucoup plus que par le passé aux phénomènes de communication. Cette priorité donnée à la communication pourrait entraîner des conséquences sur le plan de la linguistique historique qui refuse toujours d'en tenir compte suffisamment, mais elle pourrait également avoir des influences sur les théories linguistiques actuelles dont une partie considérable ne tient pas suffisamment compte des faits de communication.

A la fin de cet exposé je me vois cependant obligé d'admettre que nous n'avons pas pu atteindre l'ensemble de nos desseins : il y a des entrées auxquelles nous avons dû renoncer par manque de travaux préparatoires. Une des lacunes les plus douloureuses est celle d'une tentative de démographie linguistique historique, cela me semble constituer un besoin urgent. Il y a des langues de l'immigration pour lesquelles nous n'avons pas pu trouver le ou la spécialiste. Il y aurait quelques autres manques à relever, moins centraux et plus secondaires. Il faut également avouer aussi que toutes les contributions n'ont pas entièrement réussi à épouser nos visions. Mais, disons-le d'une manière positive : par les lacunes de notre produit nous espérons désigner des sujets pour des recherches ultérieures.

Nous espérons tout de même avoir fait un travail utile qui dans le meilleur des cas contribuera à renouveler quelque peu le regard sur certains phénomènes de la communication en France, mais également dans une perspective plus générale et ainsi peut-être servir d'exemple ${ }^{30}$.

\footnotetext{
${ }^{1}$ Je remercie François Pic de sa lecture attentive et critique de ce texte. Cependant, toutes les erreurs sont miennes.
} 
${ }^{2}$ Il est clair que l'auteur n'a pas pu établir son rapport selon des critères uniquement scientifiques, mais que des pressions politiques considérables ont dû peser sur lui. En outre, il a repris le critère de la loi Deixonne de 1951, qui ne concernait (initialement) que des langues dépourvues de statut officiel dans d'autres Etats (donc, pour ainsi dire, orphelines). Ceci peut expliquer l'intégration, dans le rapport, de langues que l'on n'y aurait peut-être pas attendu, mais aussi l'absence surprenante de certaines langues. Cf. à ce sujet: Georg Kremnitz, 2008, „Einige problematische Punkte der liste Cerquiglini“", in: Quo vadis, Romania? (Wien), no. 31, pp. 17-30.

${ }^{3}$ Bernard Cerquiglini a plus d'une fois insisté publiquement sur ce fait (qui cependant semble difficile à vérifier).

${ }^{4} \mathrm{Je}$ peux par contre affirmer de source sûre que les auteurs de la Charte avaient bien en tête la situation particulière française et ont essayé d'en tenir compte.

${ }^{5}$ Dans ce sens, j'adressai, le 12 avril 2003, une lettre à M. Bernard Cerquiglini, alors Délégué Général à la Langue Française et aux Langues de France, qui me répondit par lettre datée du 6 juin 2003. C'est de cet échange de courrier que l'initiative ici décrite prit son départ. La DGLFLF a non seulement assuré matériellement le premier colloque, mais elle a toujours veillé (notamment financièrement) à ce que le collectif puisse continuer son travail. En cela nous lui devons beaucoup.

${ }^{6}$ Malheureusement, au moment même du colloque, M. Cerquiglini quitta la direction de la DGLFLF pour une chaire à l'Université de Baton Rouge (USA). Le collectif perdit, de cette façon perdu un interlocuteur privilégié. Cependant, son successeur, M. Xavier North, nous a toujours accordé le même soutien ferme.

${ }^{7}$ Au cours des travaux, nous nous sommes rendu compte que nous avions besoin de compétences spéciales et pour cette raison agrandi légèrement le groupe initial. En outre, en 2007, Christiane Böck trouva un autre travail et dut renoncer à sa tâche.

${ }^{8}$ Cf. surtout Lukács, Georg, 1923. Geschichte und Klassenbewusstsein. Studien über marxistische Dialektik. Berlin : Malik, passim

${ }^{9}$ Winckelmann, Johann Joachim, 1764. Geschichte der Kunst des Alterthums. Dresde, 2 vols.

${ }^{10}$ Ces deux termes sont empruntés à la sociolinguistique catalane des années 1970, qui, la première a soulevé sérieusement le problème des rapports entre langues dominantes et langues dominées (en partant, bien sûr, essentiellement du cas du catalan).

${ }^{11}$ Les actes de ce colloque ont été publiés : Alén Garabato, Carmen/Boyer, Henri (dir.), 2007. Les langues de France au XXI siècle : vitalité sociolinguistique et dynamiques culturelles. Paris : L'Harmattan.

${ }^{12}$ Cf. Kremnitz, Georg, 2004. «Von der formalen Sprachwissenschaft zur Soziologie der Kommunikation“, in Das Argument (Berlin), XLVI, no. 258, pp. 809-813; id., 2005. „La linguistique du XIX ${ }^{\mathrm{e}}$ siècle, une linguistique acommunicative. Quelques réflexions », in Lengas, no. 57, pp. 111-123.

13 Cf. sur ce point les réflexions récemment publiées par Jäger, Ludwig, 2010. Ferdinand de Saussure zur Einführung, Hamburg : Junius, surtout pp. 164-190.

${ }^{14}$ Cf. de lui, p. ex. : 1918. Généalogie des mots désignant l'abeille d'après l'Atlas linguistique de la France. Paris : Champion; 1922. Les étymologies des étymologistes et celles du peuple. Paris: Champion; 1923. Thaumaturgie linguistique. Paris : Champion, etc.

${ }^{15}$ Bühler, Karl, 1934. Sprachtheorie. Die Darstellungsfunktion der Sprache. Jena: Fischer, surtout pp. 24-33.

16 Jakobson, Roman, 1960. „Linguistics and Poetics“, in Sebeok, Thomas A. (ed.), Style in Language. Cambridge/Mass.: MIT-Press, pp. 350-377.

${ }^{17}$ Shannon, Claude Elwood/Weaver, Warren, 1949. The Mathematical Theory of Communication. Urbana/Ill.: Univ. of Illinois Press.

${ }^{18}$ Je renvoie au livre jadis assez influent de Epstein, Izhac, 1915. La pensée et la polyglossie. Paris/Lausanne : Payot.

${ }^{19}$ Montesquieu le formula ainsi: « la nation, c'est-à-dire les seigneurs et les évêques », cf. p. ex. Schulze, Hagen, 1994. Staat und Nation in der europäischen Geschichte. München : Beck, p. 117.

${ }^{20}$ Pour rendre plus explicite et clair ce que nous disons.

${ }^{21} \mathrm{Il}$ y a bien sûr des histoires sociales avant la lettre, mais elles ne sont, la plupart du temps, pas faciles à dénicher. 
${ }^{22}$ P. ex.: Leith, Dick, 1983. A Social History of English. London: Routledge \& Kegan Paul [nouvelle édition en 1997, London : Routledge]; Burke, Peter/Porter, Roy (eds.), 1987. The Social History of Language. Cambridge : Cambridge Univ. Press; Jenkins, Geraint H., 1997. A Social History of the Welsh Language. Vol. I: The Welsh Language before the Industrial Revolution. Cardiff: Univ. of Wales Press; id., 2000. The Welsh Language and its Social Domains, 1801-1911. Cardiff: Univ. of Wales Press; Moreno Fernández, Francisco, 2005. Historia social de las lenguas de España. Barcelona: Ariel.

${ }^{23}$ Il n'est pas possible, dans le cadre de cette présentation, de dessiner les contours de ces évolutions. Mais je pense que cela n'est pas nécessaire ici.

${ }^{24}$ Cf. p. ex. Kremnitz, Georg, 2008. „Sur la délimitation et l'individuation des langues. Avec des exemples pris principalement dans le domaine roman », in Estudis Romànics (Barcelona), XXX, pp. 7-38.

${ }^{25}$ Dans une perspective sociolinguistique et communicative, cette dénomination tend à occulter des relations de domination politique et sociale. Dans un certain sens, toutes les langues vivantes sont "régionales » car elles s'emploient dans un ou plusieurs espaces (si l'on fait abstraction du fait que les nouvelles données de la communication tendent, au moins partiellement, à déterritorialiser toutes les langues). Ce n'est que la situation politique : statut officiel d'une langue et par conséquent possibilités communicatives limitées de toutes les autres langues qui provoque les problèmes particuliers de celles-ci. C'est pourquoi je n'emploie personnellement plus ce terme et le remplace en général par celui de langue dominée.

${ }^{26} \mathrm{Ce}$ critère n'a d'ailleurs pas été respecté entièrement: le basque et le catalan et récemment l'occitan (en Val d'Aran) sont co-officiels en Espagne, le tamazight (berbère) a entre temps été reconnu comme langue nationale au Maroc et en Algérie. Et la distinction entre arménien et arménien occidental (qui apparait dans la liste) ne semble guère pertinente du point de vue de la communication. Mais il fallait, pour d'autres raisons, que ces langues soient dans la liste.

${ }^{27}$ Une telle observation devrait pourtant être possible à partir des statistiques de migration qui existent bien.

${ }^{28} \mathrm{Si}$ au $\mathrm{XIX}^{\mathrm{e}}$ siècle et pendant la première moitié du $\mathrm{XX}^{\mathrm{e}}$ ces tendances sont des fruits de la nationalisation de la communication, depuis quelques décennies elles résultent plutôt des contradictions entre globalisation d'une part et défense identitaire de l'autre. Il me semble que les buts des mouvements de renaissances ne sont aujourd'hui plus les mêmes que par le passé : si autrefois ils cherchaient la construction d'une communauté de communication, au sens plein du terme, elles tendent aujourd'hui souvent à se contenter d'une présence testimoniale, identitaire et démarcative qui ne revendique plus, en réalité, une possibilité de communication complète (bien que les revendications affichées n'aient guère changé).

${ }^{29}$ Ceci vaut surtout pour la Guadeloupe et la Martinique, de nombreux peuples de la Guyane continuent à survivre, d'autres y ont vu le jour, tandis que la Réunion n'avait pas de peuplement avant la conquête. La population de Mayotte est essentiellement autochtone.

${ }^{30}$ Le sujet que j'ai traité ici a déjà été l'objet de plusieurs présentations. J'ai largement puisé dans ces textes ; c'est pourquoi je les énumère par la suite. En plus, ils pourront donner au lecteur qui le désire une impression des progrès de notre réflexion: G.K., 2005. «Le projet d'une Histoire Sociale des Langues de France. Présentation et questions de méthode", in www.langues-de-france.org; id., 2007. "Pour une histoire sociale des langues de France", in Langues et Cité (Paris), no. 9, p. 14 ; id., 2007a. „Zum Konzept der « Sozialgeschichte von Sprachen » und zum Projekt Histoire sociale des Langues de France“, in Quo vadis, Romania? (Wien), no. 30, pp. 5-23; id., 2008. „Le projet d'une Histoire sociale des langues de France. Pourquoi ? Comment ? » in www.langues-de-france.org; cet article doit également paraître, avec quelques mises à jour, dans la revue Pontes (Chişinău). 